Open Access

\title{
Case study: evaluation of the automation of material handling with mobile robots
}

\author{
Adriana F. Melo ${ }^{*}$ and Lindsay M. Corneal(D)
}

\author{
* Correspondence: corneall@gvsu. \\ edu \\ School of Engineering, Grand Valley \\ State University, Allendale, MI \\ 49401, USA
}

\begin{abstract}
The automation of material handling is one of the solutions that many companies are relying on to reach their goals related to productivity increment, floor space optimization, higher standards for factory's safety, and allocation of workers to valueadded activities. Therefore, the objective of this study was to evaluate the current state of the material flow of finished goods for an automotive parts supplier plant and the technology available on the market to verify if it was worthwhile to invest in material handling automation. The analysis included the use of discrete event simulation to evaluate the different layout approaches combined with the mobile robots' performance. It was proven that the tandem layout was the most beneficial approach to the analyzed plant's reality with a minimal of three robots. Improvements to the material flow and automation of the labeling process were also proposed based on the study.
\end{abstract}

\section{Introduction}

Automated guided vehicles (AGVs) first started to be used in the 1950s for manufacturing. This technology began the trend of automation of material transportation. They have proved to be reliable and efficient technological equipment for more than 50 years. However, the need of fixed routes and the minimal on-board intelligence were restricting the use of AGVs to applications that required little variability on the pickup and drop off points of materials, as well as no interruption to its path. Changes to the pathways were simply too expensive and disruptive to be cost-effective as they are typically installed in the floor.

In response to the AGV's drawbacks, a more sophisticated solution to material handling was recently introduced to the market, the autonomous mobile robots (AMRs). These robots have on-board intelligence and real-time adaptive capabilities, which support the increasing market demand for flexibility and for agility to comply with modification to products or processes [1].

In contrast with fixed route required by the AGVs, AMRs navigate via maps that its software constructs on-site or via pre-loaded facility drawings. The AMR uses data from cameras and built-in sensors and laser scanners as well as sophisticated software that enables it to detect its surroundings and choose the most efficient route to the target. To change its mission, the AMR only needs simple software adjustments. This

(c) The Author(s). 2020 Open Access This article is licensed under a Creative Commons Attribution 4.0 International License, which permits use, sharing, adaptation, distribution and reproduction in any medium or format, as long as you give appropriate credit to the original author(s) and the source, provide a link to the Creative Commons licence, and indicate if changes were made. The images or other third party material in this article are included in the article's Creative Commons licence, unless indicated otherwise in a credit line to the material. If material is not included in the article's Creative Commons licence and your intended use is not permitted by statutory regulation or exceeds the permitted use, you will need to obtain permission directly from the copyright holder. To view a copy of this licence, visit http://creativecommons.org/licenses/by/4.0/. 
functionality allows the same robot to perform a variety of different tasks at different locations, automatically adjusting to meet changing environments and production requirements. This flexibility also makes the AMRs more cost-effective as an AMR does not need wires, magnetic stripes, or other costly modifications to the building infrastructure.

Regarding the safety of these mobile systems, many of them are based on the same technology as is used for autonomous vehicles, which apply LIDAR (light detection and ranging) sensors [1]. These sensors are commonly used for pedestrian recognition. When compared with cameras, LIDARs can provide accurate range information and a larger field of view [2]. From a safety standpoint, this technology has what is recognized as a capable safety system to detect objects and people and to react appropriately [1].

It is important to emphasize, however, that new safety concerns are being raised due to this new technology. As the mobile robots are becoming more autonomous, correcting their path while in motion and the integration of new features and attachments on them, the robotics online marketing team within the Robotic Industries Association states that the existing safety standards present gaps that fail to cover some of these latest technologies to ensure the well-being of other laborers. To overcome this issue, the outlining guidelines for robot manufacturers and system integrators are being developed. The fast pace of the development of the safety standards reflects not only the urgent need for safety standards but the expectation that industrial mobile robots will continue to be implemented at a rapid rate [3].

Regarding its implementation, Gutta et al. state that facility layout design methodology is one of the primary aspects to be dealt with for an efficient and economical working of a mobile robot system. The decisions concerning material handling within the facility can have a significant impact on the effectiveness of the layout plan. Hence, for proper sequential working of a manufacturing system, it is important that the layout plan and material handling system for a facility are designed simultaneously [4].

For this study, two aspects were studied to evaluate the best way to automate the material handling at the selected plant: the plant layout and the material flow.

Facility layout design involving the implementation of mobile robots is categorized into layout design, pickup and delivery points, and flow path design. In layout design, the workspace can be seen as a set of complex polygons known as cells. The material flow system, in its turn, is characterized in terms of flow path design and direction of vehicle flow, and number and location of pickup and delivery points [5]. The flow path and location of pickup and delivery points must be determined effectively as they influence the path that a mobile robot must track, which impacts the flexibility and operational costs of a manufacturing system [6].

Typically, there are three main types of layout designs in the AMRs: single loop, tandem, and conventional layout. In a single-loop layout, AMRs travel in a unidirectional loop. When this system is used, the vehicle flow is constrained to travel only in one direction. As all the vehicles travel in the same loop, it requires less complexity in the control system. However, with this layout, the distance traveled by the vehicle might be greater when moving from one point to another, as each vehicle must travel the whole loop to visit the same point again. Another disadvantage of this kind of layout is that a vehicle breakdown can destroy the whole system [7]. 
The tandem layout overcomes some of the disadvantages presented by the singleloop layout. This layout design decomposes into non-overlapping loops, which can contain one or more vehicles. Previous studies showed that the tandem loop with multiple vehicle (TLMV) layout has out-performed single loop and tandem with single-vehicles layouts in terms of mean flow time and vehicle utilization. The main advantage of TLMV is it is less susceptible to vehicle breakdown [8].

The conventional layout design mainly includes unidirectional layout, bidirectional layout, and multi-lane hybrid layout. The main advantage of a bidirectional layout lies in the achievement of less vehicle travel time by reducing the travel distance due to the possibility of taking shortcuts and/or using a smaller layout space. However, the complexity of the control system is very high [9].

To compare these layout different approaches and to support the decision-makers within the company, this study made use of discrete simulation modeling. The design of the simulation model is subordinated to the method which is closely connected to the chosen software solution. The software used on this project was FlexSim 2018 Update 1 , and the following steps were followed for the creation of the simulation models:

1. Creation of pathways and control points for vehicle movement

2. Add the AMR process flow template

3. Adapt the process flow template to fit the scenario being analyzed

4. Develop additional programming to imitate the variability and randomness of the real world

5. Verification and validation of the created model

The devolvement of this study was focused on proposing a solution to address the shortage of labor in an automotive parts supplier's factory and to prepare the plant for the projected increase in demand in the coming years. It is important to emphasize that the automation of material handling includes not only the implementation of mobile robots but also the automation of the labeling process and the changes to the feeding system for the production cells, sorting area, receiving/shipping area, and the warehouse. To enable this, it may be necessary to change the layout of the plant.

\section{Case description-automotive parts supplier}

With the economic growth associated with the trend to automation and Industry 4.0, the company being studied has heavily invested in robotics. For the last 5 years, this company has identified the need to automate the material handling in order to meet customer demand. This decision was based on the lack of labor on the production floor, combined with the need to increase traceability of the materials and to reduce the production lead time.

As the turnover of operators is considerably high at the company, it is essential to allocate the qualified employees to more value-added activities, such as operating complex machines. Also, a lot of effort has been expended to trace material or to adjust inventory due to human errors while scanning the products or by misplacing them.

The three main goals of this project were as follows:

1. Minimize the labor required on material 
2. Improve floor space utilization to allow the installation of more production cells

3. Optimize the current material handling flow

The challenge of this application was due to the desire to implement mobile robots on the production floor rather than warehouses.

\section{Current state}

The current state analysis was focused on the finished goods and on the processes that were impacted with the automation of the material handling. There were six production cells that would be impacted with the use of mobile robots-cells SD1, BK1, BK2, DF4, DF5, and DF6-plus the sorting area and the shipping area.

Figure 1 shows the layout of the plant at the beginning of this project development. The representation of the material flow in Fig. 1 shows the material flow that is standardized. The labeling process flow was not represented there because the forklift driver has the flexibility to go to any of the labeling stations, as well as frequently the supervisor provides the label to the cell's operator when the quantities and weight are known.

Analyzing this initial layout, it could be easily verified that the flow of material of the finished goods was not linear. For example, the location of the sorting area added unnecessary travel distance. Also, SD1 was placed close to the shipping area due to its high volume. However, if the parts built there needed to be sorted, the flow was increased tremendously as they must travel to the sorting area.

It is important to highlight that the location of the production cells could not be easily modified, as they are complex-automated cells with constraints of infrastructure installation and ceiling height. Therefore, they were considered fixed locations for this analysis.

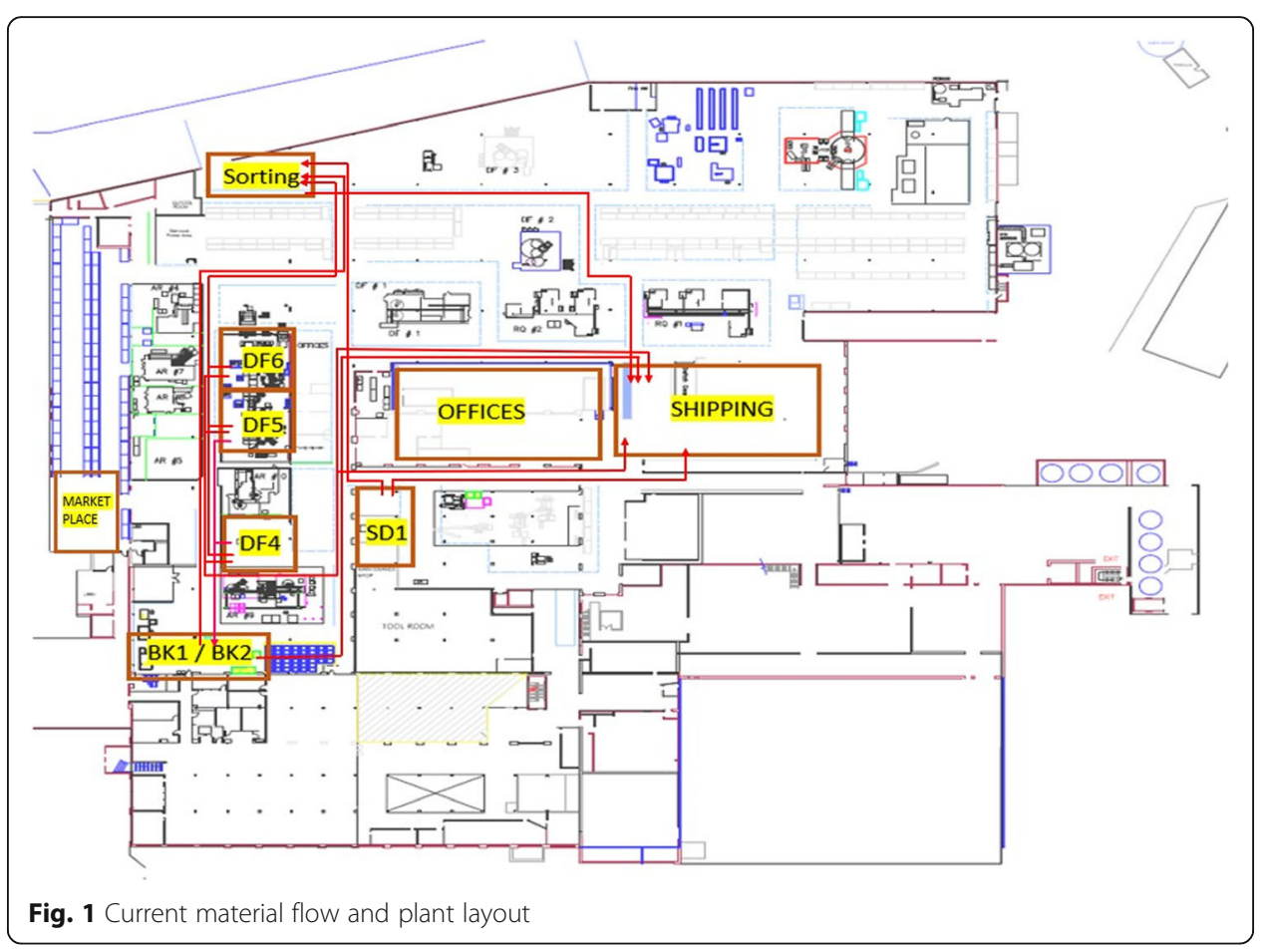


The general process for the material handling in these cells was the forklift driver brings the raw material from the receiving area to the cell. With a hand scan, the driver scans the material to execute the warehouse transfer between the previous location and its current destination. Once the parts are assembled and one tote is full, the forklift driver scans the tote and takes it to a labeling station. The tote is weighed, and a raw material (RM) label is printed. The RM label executes the transaction in the enterprise resource planning (ERP) system, which deducts the raw materials used and adds to the inventory the amount of material produced. After this label is applied to the tote, the driver takes it to its destination, which can be either the BK1 cell, the BK2 cell, the sorting area, or the shipping area. Once the tote is at its destination, the driver must scan it again, so the virtual warehouse transfer takes place.

This current process was susceptible to many errors due to its dependency on the manual labor.

\section{Layout proposal}

With the understanding of the current state of the production floor, changes were proposed to enable the automation and to improve the utilization of the mobile robots.

One way to increase the AMR efficiency was by reducing its traveling distance. Knowing that changing the location of the production cells was to be avoided, this study focused on the investigation of changes in the sorting area, shipping area, and in the labeling process.

For the sorting area, its reallocation to an area close to shipping or near to the cells which demand the most inspection was studied. Analyzing the plant layout, a solution was to move the sorting area to the market place (see Fig. 2).

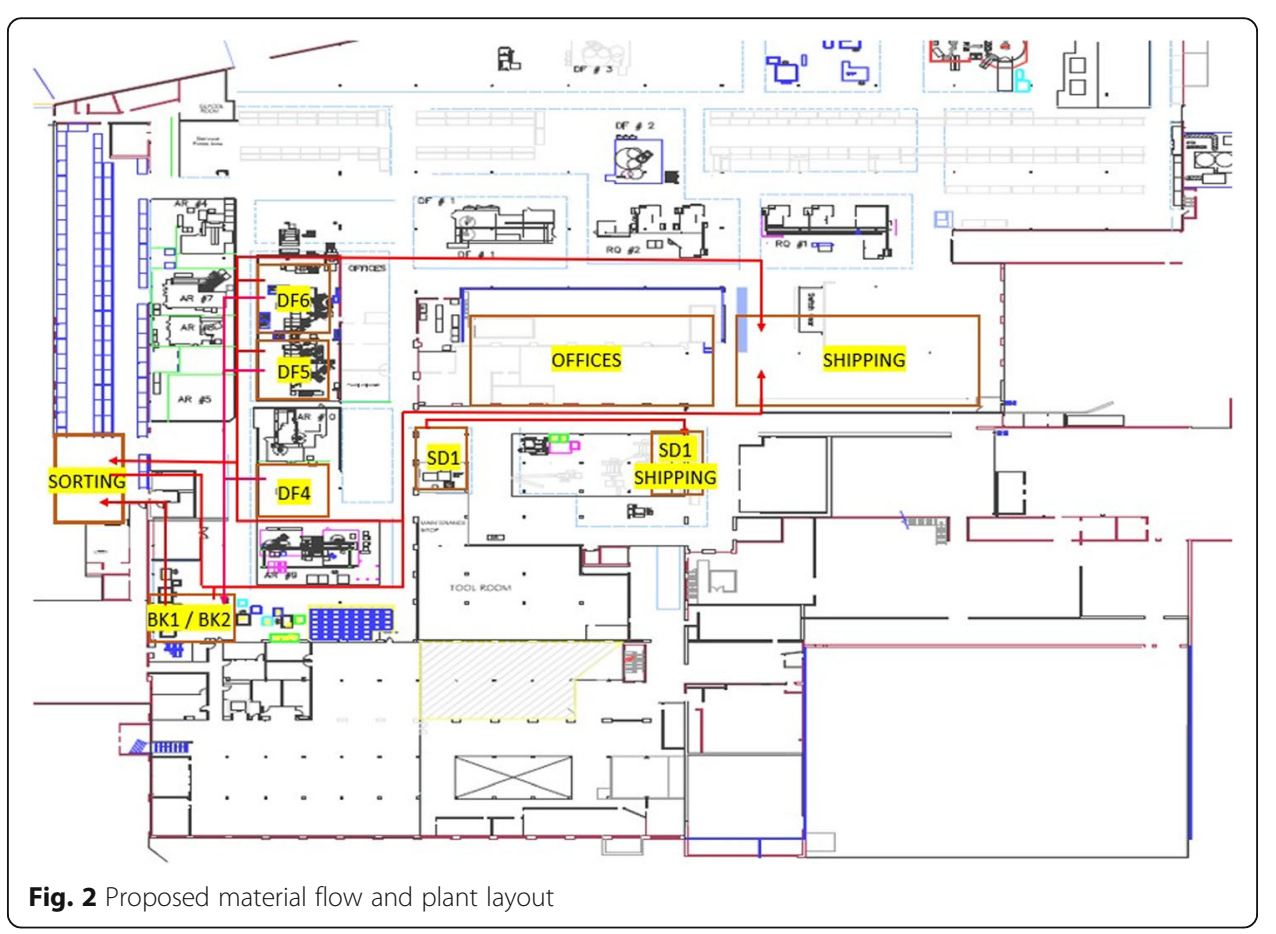


With the sorting happening at the market place, the traveling distances of the parts coming from the DF4, DF5, DF6, BK1, and BK2 would be reduced significantly. On the other hand, evaluating the parts coming from SD1, the flow of material would still not be desirable as the parts' traveling distance would more than double for the scenario when a quality inspection is required.

To solve this, it was proposed that the sorting for this cell was done in line. The high volume of SD1 supported this decision. Thus, the parts would always leave SD1 and go straight to the shipping area. Figure 2 shows the flow of material according to the changes proposed.

Regarding the shipping area, it was proposed to create an area dedicated to the SD1 parts due to the high demand. As the interaction between the mobile robots and the forklift is to be avoided, it was suggested to place the shipping area for these parts on the same side of the aisle as the cell. This would help to eliminate forklift and AMRs traveling on the same aisle in some of the layout approaches that will be discussed in the following sections. Implementing a path dedicated to the mobile robots, as well as putting in place processes that minimize the interruption of the AMRs' flow promotes the learning curve necessary when a new automation is implemented.

The end goal of the material handling automation was to have one piece flow between the production cells and the shipping area. To enable this, some enhancements were proposed to the production cells, such as the implementation of vision inspection to ensure the quality of the parts and the automation of the final packaging.

The modifications proposed in this study reduced the products lead time as they reduce their traveling distance, and decrease their cycle time by minimizing the need of manual sorting, manual labeling, and manual packaging. It also reduces the amount of labor needed.

\section{Layout scenarios}

As discussed previously, three scenarios were developed to decide which layout concept would be most appropriate for this application. The first scenario is the conventional layout, presented in Fig. 3. In this scenario, every AMR can handle the material from

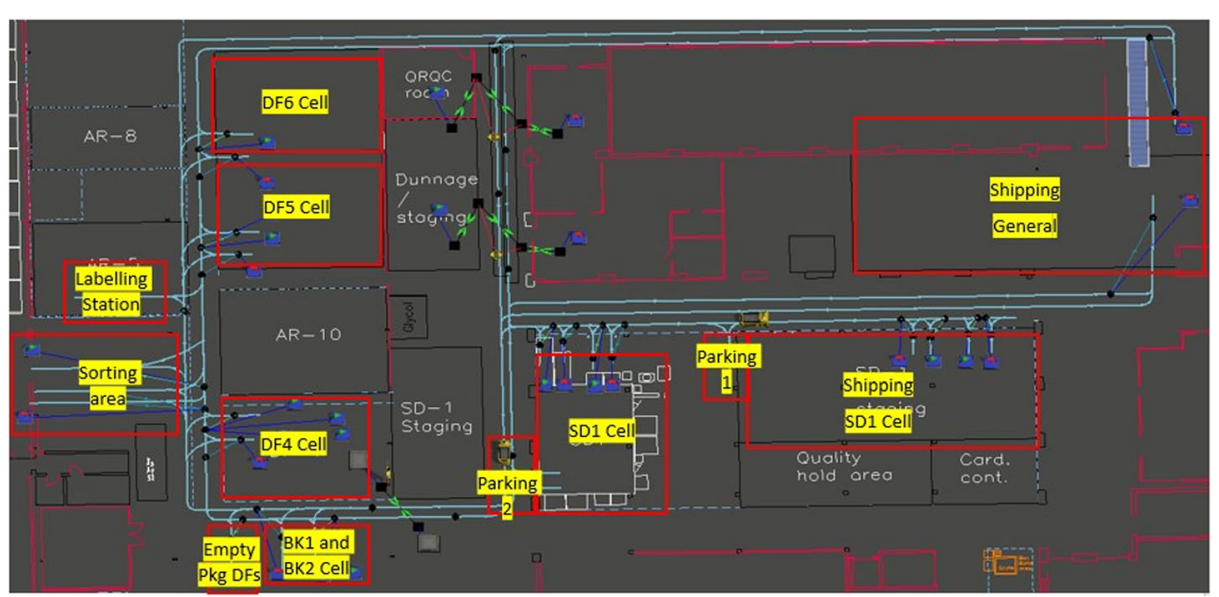

Fig. 3 Conventional layout scenario 
any production cell. This concept allows the mobile robot to judge the best route to be taken to reach its destination, and it guarantees a balanced workload between the robots. It also optimizes the priority rules, as it is applied on the whole system and not just within a section.

In addition, this concept gives more flexibility for storage location and parking spots. Also, the failure mode for this situation is less critical when compared with the other two approaches, as the tasks can be delegated to a different AMR, and the robots have more flexibility to choose a path in a blockage situation.

One of the drawbacks is that all the aisles on which the AMR will be running need to be wider to permit the passage of two AMRs on the same aisle. It requires a robust control system. This scenario also does not support the interaction avoidance between AMRs and forklifts for the SD1 cell.

The second scenario used the tandem layout, shown in Fig. 4. This approach presents a mix between the single loop concept and the conventional layout. For this simulation, the cells were divided into two sections: the first group consists of the SD1 cell and the second group handles the sorting and all the DF cells. The difference between this approach and the single loop is that more than one AMR can be placed in one section.

The tandem approach minimizes some of the drawbacks associated with the single loop (as identified below). It reduces the number of locations dedicated to storage, as all the totes for the DF cells can be put in the same place, and it permits the implementation of only one labeling station. It also gives the AMR more flexibility to select the best path to reach its destination.

This concept also allows the isolation of the SD1 cell. By putting one AMR dedicated to the SD1, a unidirectional aisle can be dedicated to the mobile robot translation which eliminates the interaction between AMRs and forklifts.

The disadvantage of the tandem layout is that if more than one AMR is allocated within one section, it necessitates wider aisles. This fact also increases the complexity of system control as the deadlock and collision situations must be addressed.

The last model followed the single loop approach, presented in Fig. 5 (3 single loops) and Fig. 6 (4 single loops). In this scenario, the plant was divided into sections where only one AMR would be responsible to handle the materials. The advantage of this

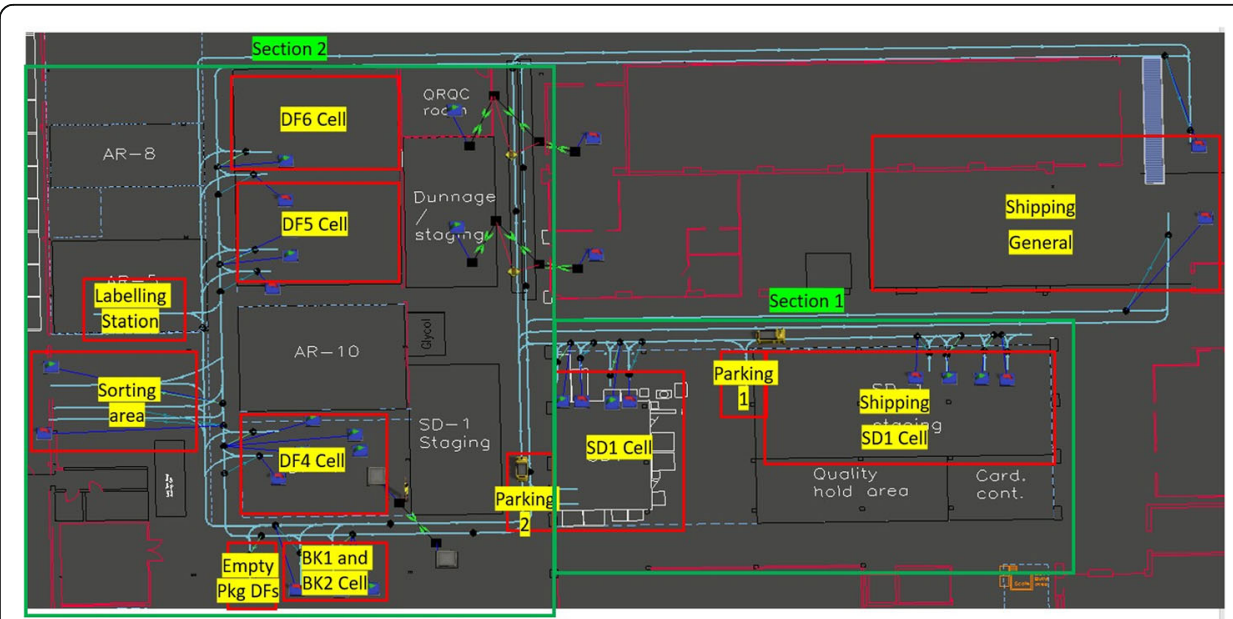

Fig. 4 Tandem layout scenario 


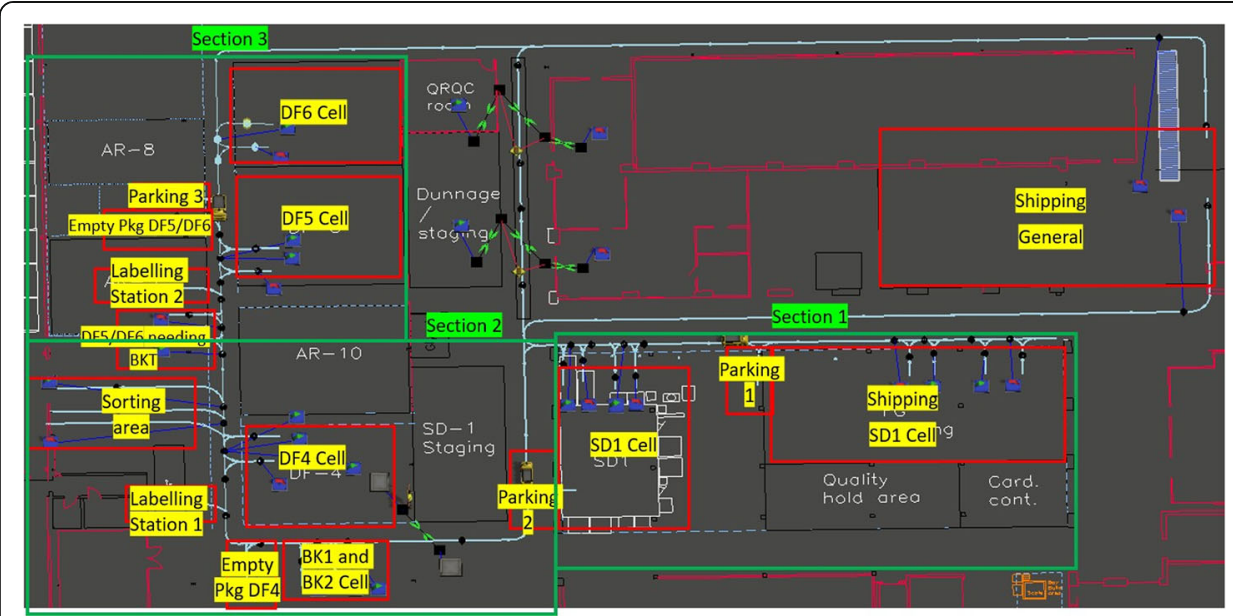

Fig. 5 Single loop layout scenario with 3 loops

solution is the reduced complexity of the control system, as the deadlock avoidance and the collisions do not need to be addressed. Also, it minimizes the width of the aisle as the AMR would not need to cross each other.

The single loop layout increases the number of storage places, parking areas, and labeling stations. It also required an intermediate location for the parts produced in DF5 and DF6 that need to go to the BK1 or BK2 cells. This scenario might also generate significant imbalanced workloads between the AMRs, and a failure mode of one robot could have a tremendous impact on the production schedule of its section. Modification on how the sections are divided would require a substantial amount of effort in programming the robots and in reallocating parking spots, storage locations, and labeling stations.

In this scenario, the AMR always follows the same path and every AMR must have a unique path.

Two options of this scenario were analyzed. In the first option, shown in Fig. 5, the production cells were divided into three sections: the first area was designated to the SD1 cell, both finished goods and brackets. The second section was for DF4 and sorting, and the third area was for DF5 and DF6. The general shipping area would have to allow the delivery of parts from both sides.

The second option was to add one more segment to separate DF5 and DF6. Thus, the workstations were divided into four sections as shown in Fig. 6. The first area was dedicated to SD1, both finished goods and brackets. The second section was dedicated to DF4. The third area was for sorting and DF5. The last one was dedicated to DF6.

It was not proposed to have a single loop with 5 divisions because it would be counterproductive due to the lack of paths to the shipping area and the high amount of intermediate storage that would be required. In both the single loop and tandem approaches, it was desired to leave SD1 by itself due to its volume and its physical separation from the rest of the cells. In all the scenarios where there is more than one AMR responsible for the material handling within a section, it was necessary to create unidirectional double paths rather than bidirectional single paths. The reason for that was the extensive blockage situations presented when single paths were applied. 


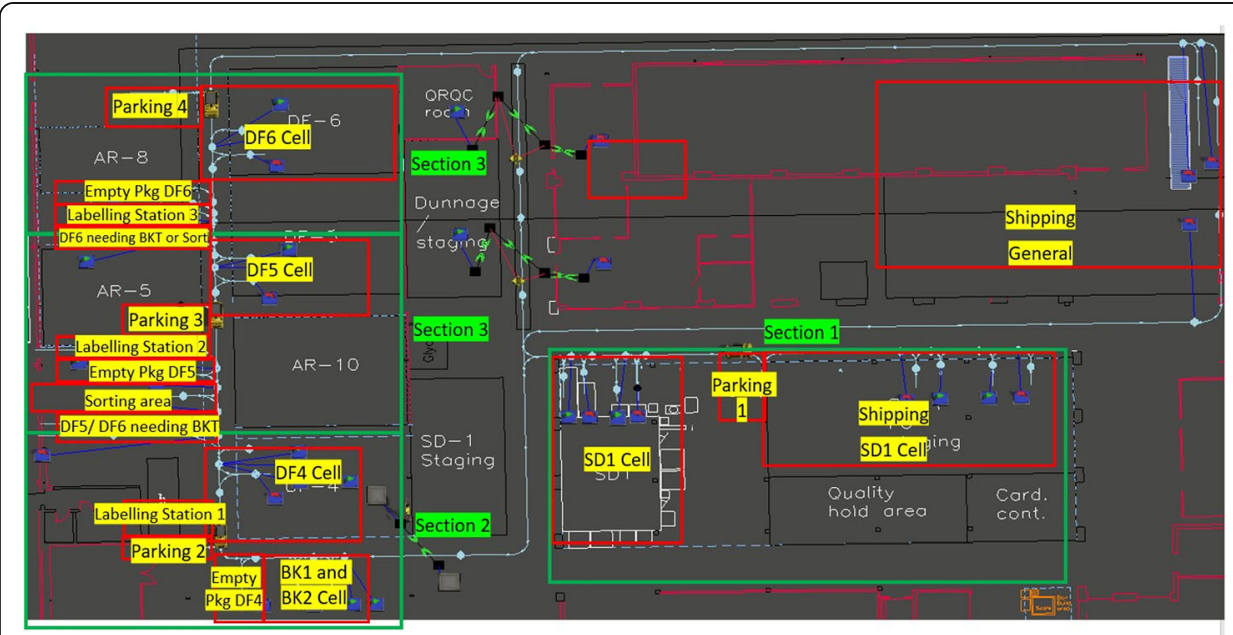

Fig. 6 Single loop layout scenario with 4 loops

\section{Comparison of scenarios}

To decide which approach would be most appropriate with the plant's reality, all three layout scenarios were simulated using FlexSim. For each layout, multiple scenarios were considered. The model parameter value that changed in each scenario was the number of mobile robots.

The performance measures were the percentage of idle time of the AMRs and the percentage of time each cell had a tote waiting for transportation. The maximum and minimal value was captured to verify the statistical dispersion of the results, as well as the confidence intervals with $99 \%$ degree of confidence. As a waiting tote can stop the cell from running, it was required that the average cells blockage be below $2 \%$.

Blockage for the DF cells means the cell is unable to produce parts due to the lack of empty totes for the new parts. A low percentage of waiting time is acceptable as the cells can manage a small buffer. However, the goal is to design a process where the wait for transportation is zero. Table 1 shows a summary of the scenarios simulated.

Also, the charging base of the AMRs was placed in their parking spots, where there was one charger for each AMR. The AMR was charging every time it was located at the parking spot. Each AMR would go to its parking spot whenever it was idle. Therefore, the amount of time the AMR was charged was equivalent to the idle time. Reviewing the specifications of the AMRs, it was decided that the AMR had to charge on average $30 \%$ of its operational time.

With respect to the demand for work, it was decided to use the production cells maximum real capacity, which accounts for machines unavailability and manufacturing errors. These measurements reduce the cells' capacity to $75 \%$ or $80 \%$ depending on the cell. This decision was based on the fact that it was completely undesired for the AMRs to be the bottleneck. For the sorting area because there are parts built in work cells other than the DF cells that need to be sorted, the data from a normal work week was used.

The average hourly demand was used with the normal distribution for internal variability of the demand estimated between 11 and $20 \%$ of the mean. The estimation of the variability was selected by analyzing 1 week of the main cells' historical data. 
Table 1 Summary of the scenarios analyzed in this study

\begin{tabular}{lll}
\hline Scenarios & & \\
\hline Layouts & Scenarios & Number of AMRs \\
\hline Conventional & Scenario 1 Conv & 1 AMR \\
& Scenario 2 Conv & 2 AMRs \\
& Scenario 3 Conv & 3 AMRs \\
& Scenario 4 Conv & 4 AMRs \\
& Scenario 5 Conv & 5 AMRs \\
& Scenario 1 Tand & 1 AMR SD1 + 1 AMR DFs \\
Tandem & Scenario 2 Tand & 1 AMR SD1 + 2 AMR DFs \\
& Scenario 3 Tand & 2 AMR SD1 + 3 AMR DFs \\
& Scenario 4 Tand & 2 AMR SD1 + 4 AMR DFs \\
Single loop—3 loops & Scenario 1 SL3 & 1 AMR SD1 + 1 AMR DF4/SORT + 1 AMR DF5/DF6 \\
Single loop—4 loops & Scenario 1 SL4 & 1 AMR SD1 + 1 AMR DF4/SORT + 1 AMR DF5 + 1 AMR DF6 \\
\hline
\end{tabular}

For safety measures, even though the majority of AMRs on the market can go up to $2 \mathrm{~m} / \mathrm{s}$, the maximum speed of all the AMRs was set to $0.8 \mathrm{~m} / \mathrm{s}$. When the robot was carrying a load, the maximum speed was reduced to $0.6 \mathrm{~m} / \mathrm{s}$. On a curved path, the speed was set to $0.6 \mathrm{~m} / \mathrm{s}$ when empty and $0.5 \mathrm{~m} / \mathrm{s}$ when loaded. The loading and unloading time were considered as $40 \mathrm{~s}$, and the labeling time was set to $60 \mathrm{~s}$. Table 2 summarizes the system's parameters.

From the results of the scenarios simulated, showed in Table 1, there were 4 scenarios that were feasible and of interest. To define if the scenario were feasible and of interest, it was analyzed using the average, the maximum, and the minimum percentage of the idle time of the robots, as well as the percentage of the cells, blocked time. Tables 3 and 4 show the overall results of each selected scenario. It also presents the confidence interval with a 99\% degree of confidence level. Comparing these results, the best layout approach was the tandem layout, where the plant was divided into two sections. The AMRs presented higher average idle time when compared with the conventional layout scenarios using 3 robots. Also, the results from scenario 4 Conv did not justify the cost of adding one more robot.

When compared with the single loop, the tandem is more beneficial due to a better workload balance between the AMRs and a better material flow.

Table 2 System's parameters for the simulations using FlexSim

\begin{tabular}{ll}
\hline Parameters of the system & \\
\hline AMRs max speed & $0.8 \mathrm{~m} / \mathrm{s}$ \\
Scenario duration & $8 \mathrm{~h}$ \\
Replication of each scenario & 20 \\
Demand for work & Normal distribuition (standard deviation: 11-20\% of the mean) \\
Warm up & No \\
Random streams & Built in on FlexSim \\
Loading time & $40 \mathrm{sec}$ \\
Unloading time & $40 \mathrm{sec}$ \\
Labeling time & $60 \mathrm{sec}$ \\
\hline
\end{tabular}




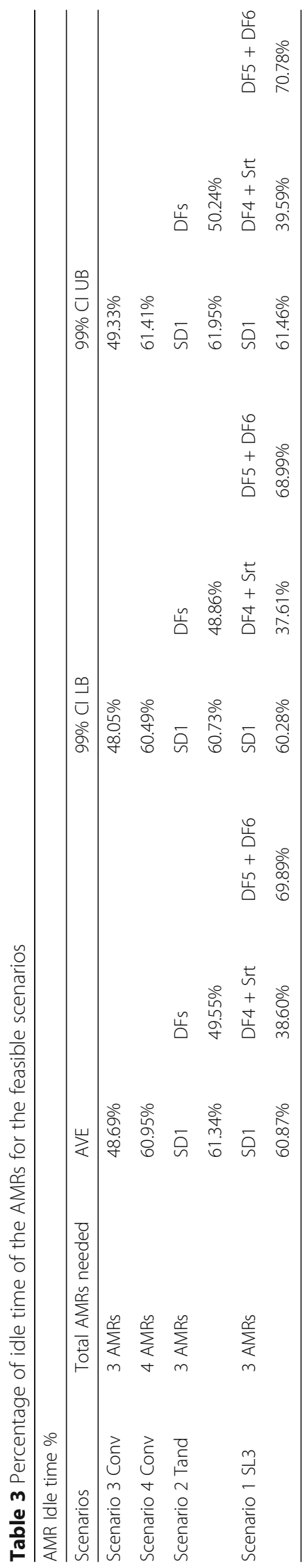




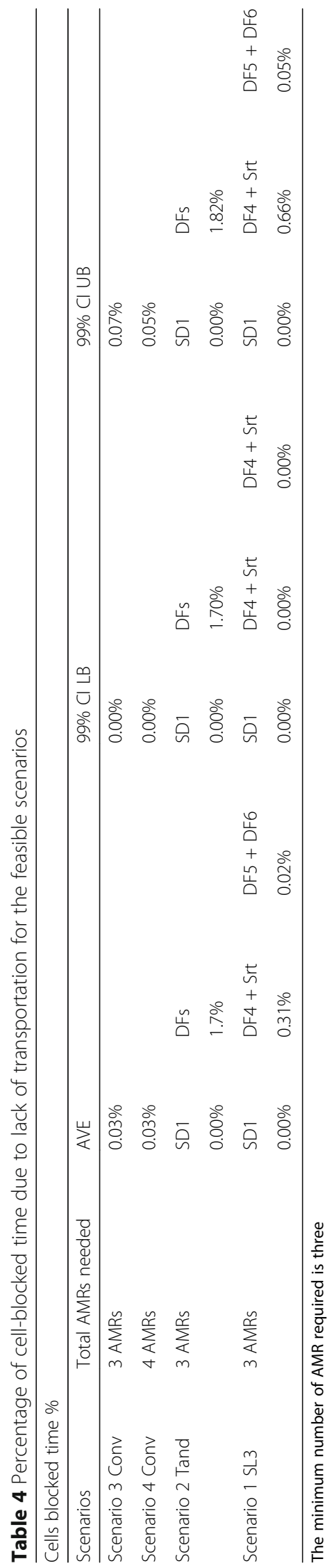




\section{Discussion and evaluation}

The key performance indicators (KPIs) that were chosen to evaluate the material handling automation system were throughput, response time, total labor dedicated to material handling, management of buffers, starvation avoidance, blocking avoidance, deadlock avoidance, dealing with disruptions, and capital cost of automation. Evaluating the chosen layout with regards to the KPIs identified, the tandem layout satisfies the throughput of the cells when 3 AMRs are used, and the starvation avoidance. This was proven by the low percentage of blockage time. This layout also provides the ability of quick response time in both sections of the system. In the DF cells section, if there is an urgency, one of the AMRs can be easily selected to execute the task. In the SD1 section, on the other hand, the AMR is dedicated to the one unique cell which will also allow for an immediate response.

Analyzing the flexibility to deal with disruptions and the blocking and deadlock avoidance, there is a tradeoff to be studied for this approach. The DF cells present a more flexible set up to deal with disruptions, as one AMR can be removed, and the cells will still be fed. However, the blockage avoidance and the deadlock avoidance need more sophisticated programming to be accomplished in this section. On the other hand, the SD1 section does not require this complex programming as there will be only one AMR operating. However, having just one AMR reduces the ability to deal with disruptions, such as breakdowns. These last two aspects need to be considered when choosing the AMR, as well as when deciding the interface between the mobile robots and the cells.

\section{Conclusions}

The next step of this project is the actual implementation. As Haneayah et al. state, the automation of the material handling is a complex installation that comprises various processes, such as inbound, storage, batching, sorting, picking, and outbound processes [10]. Therefore, it is important that the management support it as well as a culture change on the production floor.

The $5 \mathrm{~S}$ concepts are vital for the success of this project. The cells and aisles need to be kept organized, allowing the flow of the mobile robots. The blockage of aisles can have major impacts on the performance of the AMRs.

Another important point is the cooperation of the forklifts' drivers. As the material will be handled by AMRs and forklifts, the forklifts must be trained and be attentive to the AMRs to avoid blockage or damage.

With the changes required and the learning curve, it is recommended that during the first phase of the implementation the interaction between forklifts and the AMRs be avoided. This can be done by creating a separate aisle for the AMRs or creating traffic rules limiting the flow of forklifts in certain areas.

As manufacturing plants are not static places and pathways in them tend to collapse to the minimum allowable over time, this theorical study presents potential limitation regarding the adequacy for the real-world operation. However, the discrete simulation tool can be easily edited to represent the reality over time. 


\section{Acknowledgements}

The authors would like to acknowledge the contributions of Dr. Charles R. Standridge and Dr. David W. Zeitler of Grand Valley State University for their review of the analysis completed for this study.

\section{Authors' contributions}

AM prepared the simulation model, ran the simulations, and was a major contributor in writing the manuscript. LC reviewed all simulation results, provided guidance on the analysis of the results, and contributed to the writing of the manuscript. Both authors read and approved the final manuscript.

\section{Funding}

Funding for this study came from the company for which the study was completed. An author of this paper (AM) was an employee of the company for which the study was completed. The company's management had no role in the collection, analysis, or interpretation of the data.

\section{Availability of data and materials}

The data that support the findings of this study are available from the company for which the study was completed but restrictions apply to the availability of these data, and so are not publicly available. Data are however available from the authors upon reasonable request and with permission of the company for which the study was completed.

\section{Competing interests}

The author, AM, was a paid intern at the company for which this study was done. The author, LC, had no affiliation to the company.

Received: 7 February 2020 Accepted: 18 June 2020

Published online: 27 June 2020

\section{References}

1. Anandan, T. M. (2017). Industrial mobile robot safety standards on the forefront. Retrieved from https://www.robotics, org/content-detail.cfm/Industrial-Robotics-Industry-Insights/Industrial-Mobile-Robot-Safety-Standards-on-the-Forefront/ content_id/6710

2. Wang H, Wang B, Liu B, Meng X, Yang G (2017) Pedestrian recognition and tracking using 3D LiDAR for autonomous vehicle. Robotics and Autonomous Systems 88:71-78

3. Robotics Online Marketing Team. (2017). Robot safety standards for industrial mobile robots. Robotic Industries Association. Retrieved from https://www.robotics.org/blog-article.cfm/Robot-Safety-Standards-for-Industrial-MobileRobots/63.

4. Gutta PR, Chinthala VS, Manchoju RV, MVN VC, Purohit R (2017) A review on facility layout design of an automated guided vehicle in flexible manufacturing system. Material Today: Proceeding 5(2018):3981-3986

5. Farahan RZ, Pourakbar M, Miandoabchi E (2007) Developing exact and tabu search algorithms for simultaneously determining AGV loop and P/D stations in single loop systems. International Journal of Production Research 45(22): 5199-5222

6. Rajagopalan S, Heragu SS, Taylor GD (2004) A Lagrangian relaxation approach to solving the integrated pick-up/drop-off point and AGV flow path design problem. Appl Math Model 28:735-750

7. Li Q, Pogromsky A, Adriaansen T, Udding JT (2016) A control of collision and deadlock avoidance for automated guided vehicles with a fault-tolerance capability. International Journal of Advanced Robotic Systems 13(2):64

8. Ventura JA, Lee C (2001) A study of the tandem loop with multiple vehicles configuration for automated guided vehicle systems. Journal of Manufacturing Systems 20(3):153-165

9. Rajotia S, Shanker K, Batra J (1998) A heuristic for configuring a mixed uni/bidirectional flow path for an AGV system. International Journal of Production Research 36(7):1779-1799

10. Haneyah SWA, Schutten JMJ, Schuur PC, Zijm WHM (2013) Generic planning and control of automated material handling systems practical requirements versus existing theory. Computers in Industry 64:177-190

\section{Publisher's Note}

Springer Nature remains neutral with regard to jurisdictional claims in published maps and institutional affiliations.

\section{Submit your manuscript to a SpringerOpen ${ }^{\circ}$ journal and benefit from:}

- Convenient online submission

- Rigorous peer review

- Open access: articles freely available online

- High visibility within the field

- Retaining the copyright to your article

Submit your next manuscript at $\boldsymbol{\nabla}$ springeropen.com 